Revista Destaques Acadêmicos, Lajeado, v. 10, n. 3, 2018. ISSN 2176-3070

DOI: http://dx.doi.org/10.22410/issn.2176-3070.v10i3a2018.1786

http://www.univates.br/revistas

\title{
EFICÁCIA DO ÁCIDO TRANEXÂMICO NO TRATAMENTO DO MELASMA
}

\author{
Patrícia Rozo Kontze ${ }^{1}$, Paula Bianchetti ${ }^{2}$
}

Resumo: Introdução: O melasma é uma hipermelanose crônica adquirida caracterizada por manchas simétricas com tonalidade variada. A exposição solar é fator importante, mas também tem sido descrita a relação com fatores hormonais, vasculares, predisposição genética e proteínas relacionadas à tirosinase. Devido à sua natureza recorrente e refratária, o tratamento do melasma é difícil e tem como objetivo a prevenção ou a redução da área afetada, com o menor número possível de efeitos adversos. Os princípios da terapia incluem a proteção contra a radiação ultravioleta (UV) e a inibição da atividade dos melanócitos e da síntese da melanina. O ácido tranexâmico (AT) tem sido estudado como alternativa terapêutica. Objetivos: Verificar através de revisão de bibliografia a efetividade do ácido tranexâmico no tratamento do melasma. Métodos: A pesquisa foi realizada nos meses de Outubro e Dezembro de 2017, utilizando como base para a realização deste estudo, artigos e revistas científicas, pesquisados nos bancos de dados PubMed, Portal de Periódicos Capes e Google Acadêmico. Resultados: Dos artigos identificados nas bases de dados, 5 manuscritos foram incluídos para análise. Diferentes tipos de hiperpigmentações foram avaliadas nos estudos utilizando aplicação domiciliar do creme com ácido tranexâmico 3\%, injeções intradérmicas com $0,05 \mathrm{~mL}$ de ácido tranexâmico, uso oral do ácido e associação com o microagulhamento. Observou-se grau de satisfação alto por parte dos participantes dos estudos avaliados. Conclusões: O ácido tranexâmico revelou-se significativamente eficaz no tratamento do melasma, o que indica que ele é uma nova e promissora opção terapêutica. Além disso, o produto demonstrou ser seguro, dada a baixa incidência de efeitos colaterais.

Palavras-chave: Melasma. Hiperpigmentação. Ácido tranexâmico.

\section{INTRODUÇÃO}

Melasma é uma hipermelanose comum, adquirida, simétrica, caracterizada por máculas acastanhadas, mais ou menos escuras, de contornos

1 Farmacêutica, Acadêmica do curso de Pós-graduação em Estética e Saúde da Universidade do Vale do Taquari - UNIVATES, Lajeado - RS.

2 Fisioterapeuta, Doutora em Ciências Farmacêuticas (UFSM), professora da Universidade do Vale do Taquari - UNIVATES, Lajeado - RS. 
irregulares, com limites nítidos, nas áreas foto expostas, especialmente face, fronte, têmporas e, mais raramente, nariz, pálpebras, mento e membros superiores (GRIMES, 1995).

Afeta ambos os sexos, com maior incidência em mulheres, especialmente gestantes (PANDYA et al, 2006). Estudos relataram que os homens representam $10 \%$ dos casos apenas (NICOLAIDOU, KATSAMBAS, 2014). Ocorre em todas as raças, particularmente em indivíduos com fototipos altos (especialmente IV - V), que vivem em áreas com elevados índices de radiação ultravioleta (UV) (KROUMPOUZOS, COHEN, 2001).

Embora a etiologia e a patogênese do melasma não estejam completamente esclarecidas, existem vários fatores implicados. Em 30\% dos casos, a ocorrência familiar sugere predisposição genética (STEINER, FEOLA, BIALESKI, 2009).

A radiação UV é fator importante, implicado na peroxidação de lipídios na membrana celular, com liberação de radicais livres, que estimulariam os melanócitos (BOLANCA et al, 2008). Foi descrita uma relação direta entre o melasma e fatores hormonais femininos, com estudos demonstrando níveis elevados de hormônio luteinizante (LH) e baixos de estradiol sérico (HASSUN, BAGATIN, VENTURA, 2008). Além disso, a microscopia eletrônica demonstra aumento da síntese de tirosinase nas lesões de melasma (VICTOR, GELBER, RAO, 2004).

A literatura descreve três tipos de melasma: epidérmico, dérmico e misto, conforme o local de depósito deste pigmento. A maioria dos casos possui padrão misto. No epidérmico, a concentração maior de melanócitos e melanina ocorrem na camada basal e epiderme, proporciona uma coloração castanha à pele, com um aumento da melanina nos melanócitos e queratinócitos da epiderme. No melasma dérmico o pigmento encontra-se na derme, nos melanófagos. Possui diferenças de cor, variando do castanho ao azulado, às vezes até acinzentado, em razão do aumento de melanina nos macrófagos da derme.

Esta classificação tem especial importância para definir a escolha terapêutica e o prognóstico (SOUZA, GARCEZ, 2005).

De acordo com Nicolaidou E Katsambas (2014) na distribuição das manchas, são reconhecidos três padrões clínicos de melasma:

* Padrão centro facial: padrão mais comum e envolve a área da testa, bochechas, lábio superior e queixo;

* Padrão malar: acomete as regiões malares das bochechas e do nariz;

* Padrão mandibular: evolve o ramo mandibular e afeta de $9 \%$ a $16 \%$ dos casos.

Saliente-se que o melasma não deve ser visto apenas como um distúrbio orgânico, porém o paciente precisa ser avaliado na sua totalidade, levando- 
se em consideração a exposição solar, a história genética, o uso de terapias hormonais, as comorbidades, o impacto que a doença pode ter na sua vida social (MATOS, CAVALCANTI, 2009).

Um fato bem documentado na literatura, é que o melasma tem um profundo impacto na qualidade de vida de seu portador (PURIM, AVELAR, 2012). O tratamento do melasma tem como principal objetivo o clareamento das lesões e a prevenção e redução da área afetada, com o menor número possível de efeitos adversos (KIM et al, 2007).

O ácido tranexâmico (AT), que é um composto hidrofílico inibidor da plasmina, classicamente utilizada como agente antifibrinolítico, tem sido estudado como alternativa para o tratamento do melasma (MANOSROI, PODJANASOONTHON, MANOSROI, 2002). Estudos recentes revelaram que seu uso tópico previne a pigmentação induzida por UV em cobaias (MAEDA, NAGANUMA, 1998) e que seu uso intradérmico intralesional produz clareamento rápido (LEE et al, 2006).

O AT bloqueia a conversão do plasminogênio (presente nas células basais epidérmicas) em plasmina, por meio da inibição do ativador de plasminogênio. A plasmina ativa a secreção de precursores da fosfolipase A2, que atuam na produção do ácido araquidônico e induzem a liberação de fator de crescimento de fibroblasto (bFGF). Trata-se de um potente fator de crescimento de melanócito (MAEDA, TOMITA, 2007).

Já o ácido araquidônico é precursor de fatores melanogênicos, como, por exemplo, prostaglandinas e leucotrienos. O ativador de plasminogênio é gerado pelos queratinócitos e aumenta a atividade dos melanócitos in vitro. Apresenta níveis séricos aumentados com o uso de anticoncepcionais orais e na gravidez. O bloqueio dessa substância pode ser por mecanismo parácrino, por meio do qual o AT reduz a hiperpigmentação do melasma (MAEDA, TOMITA, 2007).

O composto possui propriedades antifibrinolítico, atua por um mecanismo competitivo que inibe a proteína ativadora do plasminogênio, impede a formação da plasmina, proteína responsável pela lise da fibrina, componente essencial do coágulo; tem sido utilizado para doenças de pele, tais como eczema, urticária e irritação induzida por fármacos (LI et al, 2014).

Sua atividade inibe a síntese de melanina nos melanócitos pela interferência com a interação dos melanócitos e queratinócitos através da inibição do sistema plasmina - plasminogênio; inibe a síntese de melanina não pela atuação direta nos melanócitos, mas através da inibição dos ativadores dos melanócitos contidos na cultura de queratinócitos condicionados (KARN et al, 2012).

Como contraindicação salienta-se pacientes com hemofilia e fazendo uso concomitante de complexo protrombínico ativado, devido ao risco de ocorrência de tromboembolismo (WU et al, 2012). Os efeitos adversos são 
mínimos e apresentam-se como eritema, equimose e ardência no local (TSE, HUI, 2013).

Com isso o objetivo desta pesquisa é identificar na bibliografia disponível qual a ação do Ácido Tranexâmico em melasmas.

\section{METODOLOGIA}

O estudo é uma revisão sistemática de literatura. Foram utilizados artigos científicos no idioma inglês e português consultados nas bases de dados PubMed, Portal de Periódicos Capes, utilizando as seguintes palavraschave como critério de inclusão em português: melasma, clareamento, hiperpigmentação, ácido tranexâmico, tratamento e os termos em inglês: melasma, hyperpigmentation, tranexamic acid, treatment. Foram selecionados artigos no período de 2008 a 2017. As buscas foram realizadas no período Outubro a Dezembro de 2017. Os critérios de exclusão foram artigos científicos que não se classificassem como estudo do tipo ensaio clínico randomizado ou ensaio Clínico controlado.

\section{RESULTADOS E DISCUSSÕES}

Foram identificados 24 estudos que correspondiam as palavras as chaves de busca utilizadas, destes, 4 se enquadraram nos critérios de inclusão e exclusão; dos artigos selecionados, 2 estudaram o uso tópico do AT, 1 a associação do uso tópico com via oral e 1 o uso intradérmico associado ao microagulhamento (FLUXOGRAMA 1).

Fluxograma 1 - Materiais disponíveis para a realização da revisão bibliográfica

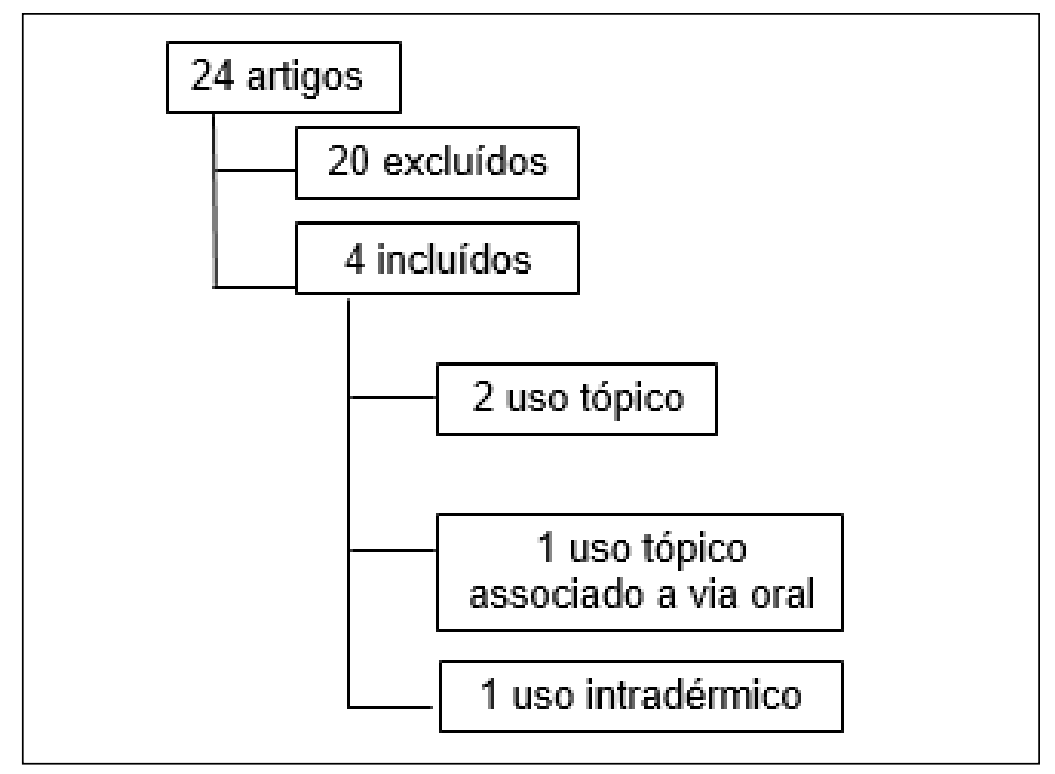


Steiner et al (2009) estudou 18 participantes do sexo feminino, com idade entre 23 e 52 anos, com diagnóstico clínico de melasma. Estas foram divididas em grupo $A$ aplicação domiciliar do creme com ácido tranexâmico 3\% duas vezes ao dia; grupo $B$ : injeções intradérmicas com $0,05 \mathrm{~mL}$ de ácido tranexâmico $(4 \mathrm{mg} / \mathrm{mL}$ ) uma vez por semana. No grupo A, houve melhora em $12,5 \%$, piora em $50 \%$ e, em $37,5 \%$, não houve alterações. No grupo B, $66,7 \%$ das pacientes apresentaram melhora, $11,1 \%$ piora, e $22,2 \%$ permaneceram inalteradas.

Em outro estudo, 23 pacientes aplicaram a fórmula de ácido tranexânico a $3 \%$ na face toda durante 12 semanas. Os pacientes apresentavam melasma moderado (índice de severidade de melasma $<5$ ). $\mathrm{O}$ resultado foi que, 22 dos 23 pacientes referiram melhora do índice de severidade do melasma após o tratamento e 10 pacientes foram submetidos à biópsia de pele a fim de avaliar vascularização e pigmentação e o que mostrou redução na quantidade de melanina na epiderme e redução no número de vasos e fator de crescimento endotelial vascular (KIM et al, 2016).

Ainda, foi realizado um estudo clínico com 25 mulheres a fim de avaliar o efeito do AT sobre o melasma. Essas mulheres ingeriram três comprimidos, três vezes ao dia, e aplicaram o AT 3\% em toda a face, duas vezes ao dia, durante oito semanas. Ao fim do estudo, observaram que houve redução da pigmentação epidérmica, assim como da vascularidade e do número de mastócitos (NA et al, 2012).

Por fim, outro estudo comparou microinjeções de AT com a associação do ácido e o microagulhamento em pacientes com melasma. Houve maiores resultados nos pacientes tratados com a associação do microagulhamento, embora a diferença não seja estatisticamente significativa (BUDAMAKUNTLA et al, 2008).

\section{CONCLUSÃO}

O estudo realizado demonstrou que os tratamentos apresentam diferentes formas de administração do AT, mas sempre devem estar associados aos hábitos de proteção contra os raios ultravioletas e ao uso de cremes despigmentantes como base fundamental no tratamento do melasma

Apesar de a avaliação clínica subjetiva ter demonstrado superioridade do tratamento injetável, na avaliação objetiva, ambos os tratamentos mostraram-se eficazes, sem diferença estatística entre os grupos. Concluindo, esse tratamento mostrou-se eficaz, sem efeitos colaterais significativos.

Concluiu-se, então, que o AT apresenta-se como uma nova e promissora opção terapêutica para o melasma, podendo ser utilizado tanto na forma de creme como de solução injetável. 


\section{REFERÊNCIAS}

BOLANCA, I. et al. Chloasma-the mask of pregnancy. Collegium Antropologicum.v. 32, n. 2, p.139-41, 2008.

BUDAMAKUNTLA, L. et al. A Randomised, Open-label, Comparative Study of Tranexamic Acid Microinjections and Tranexamic Acid with Microneedling in Patients with Melasma. Journal of Cutaneous Aesthetic Surgery, v. 6, n. 3, p. 139-43, 2013.

GRIMES, P.E. Melasma. Etiologic and therapeutic considerations. Archives of Dermatology, v.131, n. 12, p 1453-7,1995.

HASSUN, K.M.; BAGATIN, E.; VENTURA, K.F. Melasma. Revista Brasileira de Medicina, v. 65, n. 11, p. 6, 2008.

KARN, D. et al. Oral tranexamic acid for the treatment of melasma. Kathmandu University Medical Journal, v. 10, n. 40, p. 40-3, 2012.

KIM, H. et al. The vascular characteristics of melasma. Journal of Dermatology Science, v. 46, n. 2, p. 111-116, 2007.

KIM, S.J., et al. Efficacy and possible mechanisms of topical tranexamic acid in melasma. Clinical and Experiimental Dermatology, v. 41, n. 5, p. 480-5, 2016.

KROMPOUZOS, G.; COHEN, L.M. Dermatoses of pregnancy. The Journal of the American Academy of Dermatology, v. 45, p. 1-18, 2001.

LEE, J.H., et al. Localized Intradermal Microinjection of Tranexamic Acid for Treatment of Melasma in Asian Patients: A Preliminary Clinical Trial. Dermatologic Surgery, n. 32, p. 262-631, 2006.

LI, Y., et al. Treatment of melasma with oral administration of compound tranexamic acid: a preliminary clinical trial. Journal of the European Academy of Dermatology and Venereology, n. 28, v.3, p. 393-394, 2014.

MAEDA, K.; NAGANUMA, M. Topical trans-4-aminomethylcyclohexanecarboxylic acid prevents ultraviolet radiation induced pigmentation. Journal of Photochemintry and Photobiology B: Biology, v.47, p. 130-41, 1998.

MAEDA, K.; TOMITA, Y. Mechanism of the inhibitory effect of tranexamic acido $\mathrm{n}$ melanogenesis in cultured human melanocytes in the presence of keratinocyteconditioned médium. Jounal of Health Sciences, v. 53, n. 4, p.389-96, 2007.

MANOSROI, A.; PODJANASOONTHON, K.; MANOSROI, J. Development of novel topical tranexamic acid liposome formulations. The International Journal of Pharmaceutics, v. 235, p.61-70, 2002. 
MATOS, M.G.C.; CAVALCANTI, I.C. Melasma. In: Kede, M.P.V.; Sabatovich, O. Dermatologia estética. 2 ed. São Paulo: Atheneu, 2009.

NA, J.I. et al. Effect of tranexamic acid on melasma: a clinical trial with histological evaluation Journal of the European Academy of Dermatology and Venereology, v. 47, n. 2-3, p. 136-141, 2012.

NICOLAIDOU, I. E.; KATSAMBAS, A.D. Pigmentation disorders: hyperpigmentation and hypopigmentation. Clinics in Dermatology, v.32, p. 66-72, 2014.

PANDYA, A.et al. Guidelines for clinical trials in melasma. British Journal of Dermatology, v. 156, n. 1, p. 21-28, 2006.

PURIM, K.S.M.; AVELAR, M.F.S. Fotoproteção, melasma e qualidade de vida em gestantes. Revista Brasileira de Ginecologia e Obstetrícia, v.34, n.5, p. 228-34, 2012.

SOUZA, R.; GARCEZ, C.E. Temas de Medicina Estética. 5 ed. Porto Alegre: IAAM/ ASIME, 2005.

STEINER, D. et al. Study evaluating the efficacy of topical and injected tranexamic acid in treatment of melasma. Surgical \& Cosmetic Dermatology, v. 1, n. 4, p. 174-177, 2009.

STEINER, D.; FEOLA, C.; BIALESKI, N., Morais e Silva F. Tratamento do melasma: revisão sistemática Surgical \& Cosmetic Dermatology, v. 1, n. 2, p. 87-94, 2009.

TSE, T.W.; HUI, E. Tranexamic acid: an important adjuvant in the treatment of melasma. Journal of Cosmetic Dermatology, v. 12, n. 1, p. 57-66, 2013.

VICTOR, F.C.; GELBER, J.; RAO, B. Melasma: a review. Journal of Cutaneous Medicine and Surgery, v. 8, n. 2, p. 97-102, 2004.

WU, S.et al. Treatment of melasma with oral administration of tranexamic acid. Aesthetic Plastic Surgery, v. 36, n. 4, p. 964-970, 2012. 